\title{
Human exfoliated deciduous teeth and oral mucosa: promising appli- cations in tissue regeneration
}

\author{
Marta Cristaldi ${ }^{1}$, Rodolfo Mauceri ${ }^{1}$, Laura Tomasello², Giuseppe Pizzolanti ${ }^{2}$, Giovanni Zito ${ }^{5}$, Riccardo \\ Alessandro ${ }^{3,4}$, Carla Giordano ${ }^{2 *}$, Giuseppina Campisi' \\ 'Department of Surgical, Oncological and Oral Sciences, University of Palermo, Palermo, Italy \\ ${ }^{2}$ Biomedical Department of Internal and Specialist Medicine (DIBIMIS), Laboratory of Regenerative Medicine, Section of Endocrinology, Diabetology and Metab- \\ olism, University of Palermo, Palermo, Italy \\ ${ }^{3}$ Department of Biopathology and Medical Biotechnology, Section of Biology and Genetics, University of Palermo, Palermo, Italy \\ ${ }^{4}$ Institute of Biomedicine and Molecular Immunology (IBIM), National Research Council, Palermo, Italy \\ ${ }^{5}$ Advanced Technologies Network (ATeN) Center, University of Palermo, Palermo, Italy
}

Article Info

\section{Article Notes}

Received: February 20, 2018

Accepted: June 04, 2018

\section{*Correspondence:}

Dr. Carla Giordano, Biomedical Department of Internal and Specialist Medicine (DIBIMIS), Laboratory of Regenerative Medicine, Section of Endocrinology, Diabetology and Metabolism, University of Palermo, Piazza delle Cliniche 2, 90127, Palermo, Italy;

Email: carla.giordano@unipa.it

C 2018 Giordano C. This article is distributed under the terms of the Creative Commons Attribution 4.0 International License.

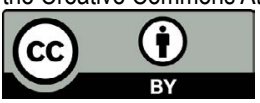

\section{Keywords}

Oral cavity

Deciduous teeth

Oral mucosa

Mesenchymal stem cells

Tissue regeneration

Pediatrics

\section{ABSTRACT}

In the last three decades, the constantly increasing need for therapies, efficiently preventing and/or treating human diseases, has raised the interest in Regenerative Medicine (RM). RM is based on employing mesenchymal stem cells (MSCs), that showed to have great proliferation, self-renewal and multilineage differentiation potential, in vitro as well as in vivo. The opportunity of an accessible, painless and low-cost reservoir of MSCs constitutes the first important step of a successful regenerative therapy to include in the current clinical practice. Oral cavity has recently demonstrated to contain different MSCs niches: dental pulp from permanent and deciduous teeth, periodontal ligament, dental follicle, apical papilla and mucosa. MSCs from dental pulp of deciduous teeth, naturally lost in pediatric age, and the oral mucosa have shown to be easily harvested and to have a promising regenerative potential. Thus, the aim of the paper is to review the potentialities of human exfoliated deciduous teeth stem cells (SHEDs) and oral mucosa stem cells (OMSCs) in RM, with the purpose of their use as accessible source of MSCs for the future of pediatric patient.

\section{Introduction}

Regenerative Medicine (RM) and Tissue Engineering (TE) are currently among the most discussed topics in medicine. Since their origin as medical branches, their goals have been to provide new strategies not only to treat diseases but also to regenerate injured tissues and organs supporting healthcare. RM and TE are based on the application of mesenchymal stem cells (MSCs), growth factors and scaffolds, artificial or natural biomaterials used as cellular support ${ }^{1,2}$. The key of the regenerative process are the properties of MSCs, multipotent cells characterized by clonogenicity, self-renewal and ability to differentiate towards different cellular lineages and regenerate various types of tissues ${ }^{3}$.

Several MSC sources have been identified as valuable sources for cellular therapy in tissue regeneration (TR). Bone marrow (BM) represents the first identified source of adult MSCs, even if the collection of BM-MSCs presents limitations due to the invasive and painful methods to harvest the samples. Moreover, even if very promising for regenerative purposes, the number, the differentiation potential and the maximal life cycle of BM-MSCs, decrease with the age of the subject ${ }^{4-6}$. Thus, other MSC sources have been investigated: the umbilical cord blood and the adipose tissue can more easily 
provide a greater number of MSCs with respect to BM by a less invasive method $^{7,8}$.

\section{Oro-facial mesenchymal stem cells}

More recently, the efforts from many researchers were focused on detecting other available sources of stem cells, leading to the characterization of the oro-facial MSCs. Many studies widely demonstrated the promising in vitro and in vivo TR potential of the oral MSCs. Noteworthy, the oral cavity contains different "niches" of stem cells: dental pulp from permanent and deciduous teeth, periodontal ligament, dental follicle, apical papilla and the three subtypes of mucosa: the masticatory mucosa, namely hard palate and gingiva; the specialized mucosa, represented by the dorsal surface of the tongue, and the lining mucosa, which includes buccal mucosa, the internal surface of the lips, the ventral surface of the tongue, the soft palate, and the alveolar mucosa ${ }^{9-16}$.

Among the oro-facial MSCs, human exfoliated deciduous teeth stem cells (SHEDs) and oral mucosa stem cells (OMSCs) have recently attracted the attention of many studies focused on TR therapies, for several reasons (See Table 1) ${ }^{9-13,17}$ :

1. the surgical procedures necessary to harvest the MSC are easy and painless;

2. the MSC isolation methods are simple, fast and lowcost;
3. as all recognized SCs, they express the canonical surface markers (e.g. CD29, CD73, CD105, CD44, CD146, CD49c, SSEA-4 and Stro-1) and nuclear markers (e.g. NANOG, Oct-4 and SOX2);

4. they show a great self-renewal ability and multilineage differentiation potential.

It has been widely demonstrated that SHEDs and OMSCs, besides the expression of canonical MSC surface and nuclear markers without any expression of hematopoietic stem cell markers, in presence of specific growth factors, differentiate towards different lineages (e.g. osteocytes, chondrocytes and adipocytes), also expressing the related specific lineage markers ${ }^{9}, 11-13,17$. Moreover, they are considered a very homogeneous population, with even a greater TR potential with respect to BM-MSCs ${ }^{9,18-21}$.

The investigations on stem cell properties of oro-facial MSCs were recently extended to inflamed tissues, showing as MSCs deriving from inflamed tissue have similar regenerative properties as the healthy counterpart, in terms of stem cell marker expression, proliferation and multilineage differentiation abilities. Alongi et al demonstrated that the human dental pulp stem cells (hDPSCs) from inflamed tissue have no difference in the MSC profiles if compared to control tissue-derived hDPSCs. Even if some stem cell properties may be affected and altered under inflammation condition, most of them remained potent in

Table 1: OMSCS and SHEDs in vitro and in vivo stem cell properties

\begin{tabular}{|c|c|c|c|c|}
\hline Ref/Author/Year & MSCs & ESC and MSC markers & In vitro regeneration & In vivo regeneration \\
\hline Miura et al (2003) [10] & SHEDs & STRO-1, CD146 & $\begin{array}{l}\text { Neurons, adipocytes and } \\
\text { odontoblasts }\end{array}$ & Bone and dentin \\
\hline Zhang et al (2009) [13] & OMSCs & $\begin{array}{l}\text { Oct-4/SSEA-4, Oct-4/STRO-1, CD29, } \\
\text { CD73, CD90/Thy-1 and CD105 }\end{array}$ & Neurons, adipocytes and osteoblasts & Connective tissue \\
\hline Wang et al (2012) [17] & SHEDs & STRO-1, CD146, CD29 and CD105 & Osteocytes and adipocytes & Bone \\
\hline Tomasello et al (2017) [22] & OMSCs & STRO-1, CD146, Cd29 and SSEA-4 & Osteocytes & - \\
\hline $\begin{array}{l}\text { Marynka-Kalmani et al (2010) } \\
\text { [25] }\end{array}$ & OMSCs & $\begin{array}{l}\text { Oct-4, Sox-2, SSEA-4, CD29, CD73, } \\
\text { CD90, CD105 and CD166 }\end{array}$ & $\begin{array}{l}\text { Neurons, adipocytes, chondrocytes } \\
\text { and osteocytes }\end{array}$ & $\begin{array}{l}\text { Bone, fat, striated } \\
\text { muscle and neural } \\
\text { tissue }\end{array}$ \\
\hline Zhang et al (2012) [26] & OMSCs & CD73, CD90, CD44, CD105 & $\begin{array}{l}\text { Adipocytes, chondrocytes and } \\
\text { osteocytes }\end{array}$ & Bone \\
\hline Machado et al (2012) [30] & SHEDS & CD29, CD73 and CD105 & Osteocytes and odontoblasts & Bone/dentin \\
\hline Nakamura et al (2009) [31] & SHEDS & CD29, CD73, CD105 and CD44 & - & - \\
\hline Nakajima et al (2018) [36] & SHEDS & CD29, CD44, CD73, CD105, CD146 & Osteocytes & Bone \\
\hline Ginani et al (2018) [37] & SHEDS & $\mathrm{CD} 90, \mathrm{CD} 73, \mathrm{CD} 105$ & Osteocytes, adipocytes & - \\
\hline Huang et al (2018) [39] & SHEDS & CD90, CD73, Oct4 & Osteocytes & 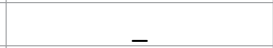 \\
\hline Tang et al (2011) [41] & OMSCs & $\begin{array}{l}\text { CD90, CD29, CD44, CD105, CD146, } \\
\text { STRO-1 }\end{array}$ & $\begin{array}{l}\text { Osteocytes, adipocytes and } \\
\text { chondrocytes }\end{array}$ & Connective tissue \\
\hline $\begin{array}{l}\text { Treves-Manusevitz et al (2013) } \\
\text { [43] }\end{array}$ & OMSCs & CD29, CD73, CD90, CD105, CD166 & $\begin{array}{l}\text { Osteocytes, adipocytes and } \\
\text { chondrocytes }\end{array}$ & Bone \\
\hline Mitrano et al (2010) [44] & OMSCs & CD90, CD105, CD73, CD44, CD13 & $\begin{array}{l}\text { Osteocytes, adipocytes and } \\
\text { chondrocytes }\end{array}$ & - \\
\hline Rajan et al (2017) [45] & OMSCs & $\begin{array}{l}\text { CD29, CD90, CD73, CD105, CD44, } \\
\text { Oct4, SOX-2, SSEA-4 }\end{array}$ & $\begin{array}{l}\text { Osteocytes, adipocytes and } \\
\text { chondrocytes }\end{array}$ & - \\
\hline
\end{tabular}

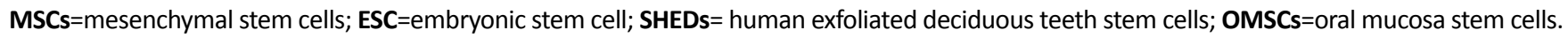


self-renewal and multi-lineage differentiation in vitro as well as in vivo ${ }^{22,23}$.

The demonstrated great potential of SHEDs and OMSCs $^{16,17,24-26}$ and the relatively easy collecting and harvesting method, led us to suggest the opportunity to use the deciduous teeth, naturally lost in pediatric age, and/or the oral mucosa, for TR purposes of patients.

For this reason, the aim of this paper is to review and clarify the regeneration potential of SHEDs and OMSCs.

\section{Deciduous teeth and oral mucosa: two promising reservoir of mesenchymal stem cells for the presence and the future of pediatric patient}

Easily accessible and with a great regenerative ability, SHEDs are valuable candidate progenitors for $\mathrm{TR}^{10,27}$. The natural intrinsic mechanism involving the transition from deciduous to permanent teeth makes them a reliable source of MSCs, eventually employable in the future of pediatric patient. Various studies showed that, as DPSCs, SHEDs have not only interesting in vitro and in vivo TR potential, being able to repair bone, cartilage, blood vessels and adipose tissue, but also a higher proliferation rate, increased population doubling and stemness properties for longer periods, compared to DPSCs and BM-MSCs ${ }^{28-31} 17$, 32,33 . These properties make them a simple, straightforward and convenient source of stem cells for treatment of many current or potential diseases of the pediatric patient, with minimal or no trauma ${ }^{32-34}$

Nakajima et al. compared bone tissue regeneration of SHEDs with that of DPSCs and human bone marrow stem cells (hBMSCs) by micro-computed tomography and histological analysis, demonstrating that SHEDs have equal bone formation ability to hBMSCs and DPSCs, and produce larger osteoids and wider distribution of collagen fibers than other sources, resulting in an efficient capability of bone defect regeneration ${ }^{35}$.

More recent studies also focused on their potential neuroprotector effects, showing that SHEDs, after transplantation in a spinal cord injury murine model, were able to induce anti-apoptotic factor activity, resulting in the neuroprotective effects on moto-neurons ${ }^{36}$.

Finally, it was also demonstrated that the proliferation and the differentiation ability of SHEDs may be enhanced by chemical and physical treatments. Two recent studies showed that physical treatments, as laser irradiation, are able to enhance the in vitro proliferation of SHEDs ${ }^{37,38}$. Moreover, in presence of specific factors, SHEDs showed higher proliferation and differentiation ability: chitosan scaffolds, functionalized with divalent metal phosphates, enhance the osteo-differentiation ability of SHEDs ${ }^{39}$. Araùjo et al. analysed the effects of mineral compounds on the regenerative properties of SHEDs and demonstrated a proliferative and differentiation advantage ${ }^{40}$. The studies above described open up to the possibility of a regenerative procedure based on SHEDs, supported or not by specific physical or chemical factors.

If on one side it is worth discussing about SHEDs TR potential, on the other side, it is also important talking about the surprising properties of OMSCs. As described above, the human oral mucosa is divided in the masticatory, the specialized and the lining mucosa ${ }^{16}$. Many laboratories have worked up-to-now to analyze and characterize the different stem cell populations, contained in the oral mucosa, by the classical MSCs hallmarks as self-renewal, in vitro and in vivo multi-lineage differentiation and expression of typical stem cell markers ${ }^{16}$.

Izumi et al. successfully isolated and characterized an oral keratinocyte-enriched population of progenitor-stem cells: able to in vitro form colonies, they ex vivo regenerate an oral mucosa equivalent ${ }^{24}$.

Also Marynka-Kalmani et al. identified in oral connective tissue (i.e. the lamina propria) another promising source of stem cells; the population of SCs was heterogeneous: part of it expressed embryonic stem cell transcription factors as Oct4, SOX2 and SSEA-4 part of it expressed the canonical MSC markers as CD29, CD73, CD90, CD105, CD166 and Stro1. They were able to differentiate towards mesodermal and ectodermal lineages ${ }^{25}$.

Many recent works also focused on investigating the regenerative properties of cells isolated from gingival tissue. Gingival mesenchymal stem cells (GMSCs) have been demonstrated to express MSCs markers, to have a high clonogenic and proliferation ability and, when in specific growth condition, show multipotency differentiation ability into several cellular lineages. They, indeed, resulted positive for MSCs markers as CD90, CD105, CD73, CD44 and CD13, and negative for hematopoietic markers as CD34, CD38, CD45 and CD54 and in presence of specific growth factors, they differentiated towards osteoblasts, adipocytes and chondroblasts, confirmed by the expression of osteogenic markers (i.e. bone sialoprotein, alkaline phosphatase and osteocalcin), adipogenic markers (i.e. peroxisome proliferator-activated receptor $\gamma$ ) and chondrogenic markers (i.e. collagen 2A1 and aggrecan) ${ }^{41-44}$.

Noteworthy, neuroprotection and immune-modulation of GMSCs were recently investigated. The conditioned medium by human GMSCs is able to increase the levels of neuroprotective factors (e.g. neurotrophins and antiinflammatory cytokines) resulting in inhibition of apoptosis after injury and neuroprotection ${ }^{45}$. GMSCs are able to inhibit proliferation of peripheral blood lymphocytes, increasing the expression of anti-inflammatory factors; indeed, the infusion of human GMSCs in an experimental model of colitis decreases the resulting effects of inflammation 
(e.g. colon inflammation, weight loss, injury on intestinal mucosa $)^{13}$.

In conclusion, even if more studies will be necessary to make SHEDs and OMSCs available for clinical use, the up-tonow knowledge about their TR potential is encouraging and represents a hopeful starting point for future therapeutic treatments.

\section{Concluding remarks}

Regenerative medicine is a constantly evolving interdisciplinary field, deriving from the collaboration of different scientific figures as biologists, clinicians, technologists, chemists, nanotechnologists that cooperate together to develop procedures aimed to manipulate and control cells and the relative surrounding environment, for TR purposes ${ }^{2}$. The need of therapies in all different biomedical areas and the promising properties of the adult MSCs led to the real possibility of successful therapies able to treat human diseases, in some cases otherwise not treatable, and this represents an attracting perspective for the future of medicine. After years of studies focused on BM-MSCs and and adipose tissue mesenchymal stem cells (AT-MSCs), which have well-known great regeneration potential, but whose harvesting and isolation procedures implicate high costs and painful clinical practices, the aim of many studies is now more and more focused on developing an easier, painless and more economic procedure. Oral cavity has been identified as a rich and more accessible reservoir of MSCs. Noteworthy, oral mucosa and pulp tissue from deciduous teeth, naturally lost in pediatric age, represent a promising and accessible source of MSCs.

Nevertheless, even if promising candidates for regenerative therapies, there are also some limitations of the above described MSCs use in clinical practice. The MSCs in vitro cell culture needs fetal bovine serum (FBS) that provides nutrients to cells. However, FBS addition could affect the safeness of MSCs for clinical use, since it could result toxic for patients. A recent study shows a little difference in SC marker expression and differentiation ability of DPSCs and BM-MSCs, grown in serum/xenofree medium ${ }^{46}$. Thereby, it is necessary to develop a safe and qualified protocol that both allowed MSC growth and expansion enough to have a good amount of cells for therapies, and ensured the safeness of cell application in clinics; it is the base of a safe translational research.

Another limitation of oro-facial MSC clinical use is the availability of cells throughout the whole patient's life. For instance, SHEDs need to be cryo-preserved until the adult age of patient; but a valuable cryo-preservation protocol is still under investigation ${ }^{47-49}$. Instead, this is not a limitation of OMSCs since the mucosa is always easily accessible and available, and there is no necessity of long-term preservation.
Finally, the lack of a standardized protocol for clinical application and the scarcity of human clinical trials evaluating the pros and cons of the use of oro-facial MSCs still limit their employment in clinical practice.

Many studies are still necessary to develop a safe protocol for oro-facial MSC clinical use; however, the upto-now results represent the encouraging idea of an everavailable ready-to-use autologous reservoir of MSCs to use for treatment of any disease occurring during the childhood (e.g. burns, orthopaedic disorders, spinal cord dysfunction, hypospadias, physeal injuries and genetic diseases as bullous epidermolysis) or adult age, without need of additional surgical sites or economic cost, once everyone naturally lost milk teeth.

\section{Financial disclosure/Acknowledgements}

Marta Cristaldi, PhD student, is supported, for this research, by the Italian Ministry of University and Research (PON Industrial PhD 2016-2017 - prot. DOT1320875), Doctoral Course of Experimental Oncology and Surgery, Cycle XXXII.

\section{Abbreviations}

AT-MSC Adipose tissue mesenchymal stem cell

BM Bone Marrow

BM-MSC Bone marrow mesenchymal stem cell

CD Cluster of differentiation

DPSC Dental pulp mesenchymal stem cell

FBS Fetal bovine serum

GMSC Gingival mesenchymal stem cell

hBMSC Human bone marrow stem cell

hDPSC Human dental pulp mesenchymal stem cell

MSC Mesenchymal stem cell

NANOG Homeobox protein NANOG

OCT4 Octamer-binding transcription factor 4

OMSC Oral mucosa stem cell

RM Regenerative medicine

SC Stem cell

SHED Human exfoliated deciduous teeth stem cell

SOX-2 SRY (sex determining region Y)-box 2

SSEA-4 Stage-specific embryonic antigen-4

TE Tissue engineering

TR Tissue regeneration 


\section{Conflicts of interest}

The authors declare that there is no conflicts of interest that could be perceived as prejudicing the impartiality of the research reported.

\section{References}

1. Ballini, Scacco, Coletti, et al. Mesenchymal Stem Cells as Promoters, Enhancers, and Playmakers of the Translational Regenerative Medicine. Stem Cells Int. 2017; 2017: 3292810.

2. Gomes, Rodrigues, Domingues, et al. Tissue Engineering and Regenerative Medicine: New Trends and Directions-A Year in Review. Tissue Eng Part B Rev. 2017; 23: 211-24.

3. Gimble, Katz, Bunnell. Adipose-derived stem cells for regenerative medicine. Circ Res. 2007; 100: 1249-60.

4. Mueller, Glowacki. Age-related decline in the osteogenic potential of human bone marrow cells cultured in three-dimensional collagen sponges. J Cell Biochem. 2001; 82: 583-90.

5. Nishida, Endo, Yamagiwa, et al. Number of osteoprogenitor cells in human bone marrow markedly decreases after skeletal maturation. J Bone Miner Metab. 1999; 17: 171-7.

6. Stenderup, Justesen, Clausen, et al. Aging is associated with decreased maximal life span and accelerated senescence of bone marrow stromal cells. Bone. 2003; 33: 919-26.

7. Zuk, Zhu, Ashjian, et al. Human adipose tissue is a source of multipotent stem cells. Mol Biol Cell. 2002; 13: 4279-95.

8. Kern, Eichler, Stoeve, et al. Comparative analysis of mesenchymal stem cells from bone marrow, umbilical cord blood, or adipose tissue. Stem Cells. 2006; 24: 1294-301.

9. Kawashima. Characterisation of dental pulp stem cells: a new horizon for tissue regeneration. Arch Oral Biol. 2012; 57: 1439-58.

10. Miura, Gronthos, Zhao, et al. SHED: stem cells from human exfoliated deciduous teeth. Proc Natl Acad Sci U S A. 2003; 100: 5807-12.

11. Seo, Miura, Gronthos, et al. Investigation of multipotent postnatal stem cells from human periodontal ligament. Lancet. 2004; 364: 14955 .

12. Sonoyama, Liu, Yamaza, et al. Characterization of the apical papilla and its residing stem cells from human immature permanent teeth: a pilot study. J Endod. 2008; 34: 166-71.

13. Zhang, Shi, Liu, et al. Mesenchymal stem cells derived from human gingiva are capable of immunomodulatory functions and ameliorate inflammation-related tissue destruction in experimental colitis. J Immunol. 2009; 183: 7787-98.

14. Egusa, Sonoyama, Nishimura, et al. Stem cells in dentistry--part I: stem cell sources. J Prosthodont Res. 2012; 56: 151-65.

15. Gronthos, Mankani, Brahim, et al. Postnatal human dental pulp stem cells (DPSCs) in vitro and in vivo. Proc Natl Acad Sci U S A. 2000; 97: 13625-30.

16. Jones, Klein. Oral epithelial stem cells in tissue maintenance and disease: the first steps in a long journey. Int J Oral Sci. 2013; 5: 121-9.

17. Wang, Sha, Li, et al. Comparative characterization of stem cells from human exfoliated deciduous teeth and dental pulp stem cells. Arch Oral Biol. 2012; 57: 1231-40.

18. Hallman, Thor. Bone substitutes and growth factors as an alternative/ complement to autogenous bone for grafting in implant dentistry. Periodontol 2000. 2008; 47: 172-92.

19. Kolk, Handschel, Drescher, et al. Current trends and future perspectives of bone substitute materials - from space holders to innovative biomaterials. J Craniomaxillofac Surg. 2012; 40: 706-18.
20. Alge, Zhou, Adams, et al. Donor-matched comparison of dental pulp stem cells and bone marrow-derived mesenchymal stem cells in a rat model. J Tissue Eng Regen Med. 2010; 4: 73-81.

21. Yu, Wang, Deng, et al. Odontogenic capability: bone marrow stromal stem cells versus dental pulp stem cells. Biol Cell. 2007; 99: 465-74.

22. Tomasello, Mauceri, Coppola, et al. Mesenchymal stem cells derived from inflamed dental pulpal and gingival tissue: a potential application for bone formation. Stem Cell Res Ther. 2017; 8: 179.

23. Alongi, Yamaza, Song, et al. Stem/progenitor cells from inflamed human dental pulp retain tissue regeneration potential. Regen Med. 2010; 5: 617-31.

24. Izumi, Tobita, Feinberg. Isolation of human oral keratinocyte progenitor/stem cells. J Dent Res. 2007; 86: 341-6.

25. Marynka-Kalmani, Treves, Yafee, et al. The lamina propria of adult human oral mucosa harbors a novel stem cell population. Stem Cells. 2010; 28: 984-95.

26. Zhang, Nguyen, Yu, et al. Human oral mucosa and gingiva: a unique reservoir for mesenchymal stem cells. J Dent Res. 2012; 91: 1011-8.

27. Demarco, Conde, Cavalcanti, et al. Dental pulp tissue engineering. Braz Dent J. 2011; 22: 3-13.

28. Govindasamy, Ronald, Totey, et al. Micromanipulation of culture niche permits long-term expansion of dental pulp stem cells--an economic and commercial angle. In Vitro Cell Dev Biol Anim. 2010; 46: 764-73.

29. Rosa, Della Bona, Cavalcanti, et al. Tissue engineering: from research to dental clinics. Dent Mater. 2012; 28: 341-8.

30. Machado, Fernandes, Gomes Pde. Dental stem cells for craniofacial tissue engineering. Oral Surg Oral Med Oral Pathol Oral Radiol. 2012; 113: 728-33.

31. Nakamura, Yamada, Katagiri, et al. Stem cell proliferation pathways comparison between human exfoliated deciduous teeth and dental pulp stem cells by gene expression profile from promising dental pulp. J Endod. 2009; 35: 1536-42.

32. Arora, Arora, Munshi. Banking stem cells from human exfoliated deciduous teeth (SHED): saving for the future. J Clin Pediatr Dent. 2009; 33: 289-94.

33. Gioventu, Andriolo, Bonino, et al. A novel method for banking dental pulp stem cells. Transfus Apher Sci. 2012; 47: 199-206.

34. Nishino, Yamada, Ebisawa, et al. Stem cells from human exfoliated deciduous teeth (SHED) enhance wound healing and the possibility of novel cell therapy. Cytotherapy. 2011; 13: 598-605.

35. Nakajima, Kunimatsu,Ando, etal. Comparison of the bone regeneration ability between stem cells from human exfoliated deciduous teeth, human dental pulp stem cells and human bone marrow mesenchymal stem cells. Biochem Biophys Res Commun. 2018; 497: 876-82.

36. Nicola, Marques, Odorcyk, et al. Neuroprotector effect of stem cells from human exfoliated deciduous teeth transplanted after traumatic spinal cord injury involves inhibition of early neuronal apoptosis. Brain Res. 2017; 1663: 95-105.

37. Ginani, Soares, de Oliveira Rocha, et al. Low-level laser irradiation induces in vitro proliferation of stem cells from human exfoliated deciduous teeth. Lasers Med Sci. 2018; 33: 95-102.

38. Vale, Maria, Picoli, et al. The Effects of Photobiomodulation Delivered by Light-Emitting Diode on Stem Cells from Human Exfoliated Deciduous Teeth: A Study on the Relevance to Pluripotent Stem Cell Viability and Proliferation. Photomed Laser Surg. 2017; 35: 659-65.

39. Huang, Su, Chen. Comparing the Effects of Chitosan Scaffolds Containing Various Divalent Metal Phosphates on Osteogenic Differentiation of Stem Cells from Human Exfoliated Deciduous Teeth. Biol Trace Elem Res. 2018. 
40. Araujo, Cosme-Silva, Fernandes, et al. Effects of mineral trioxide aggregate, BiodentineTM and calcium hydroxide on viability, proliferation, migration and differentiation of stem cells from human exfoliated deciduous teeth. J Appl Oral Sci. 2018; 26: e20160629.

41. Tang, Li, Xie, et al. Characterization of mesenchymal stem cells from human normal and hyperplastic gingiva. J Cell Physiol. 2011; 226: 832-42.

42. Fournier, Larjava, Hakkinen. Gingiva as a source of stem cells with therapeutic potential. Stem Cells Dev. 2013; 22: 3157-77.

43. Treves-Manusevitz, Hoz, Rachima, et al. Stem cells of the lamina propria of human oral mucosa and gingiva develop into mineralized tissues in vivo. J Clin Periodontol. 2013; 40: 73-81.

44. Mitrano, Grob, Carrion, et al. Culture and characterization of mesenchymal stem cells from human gingival tissue. J Periodontol. 2010; 81: 917-25.

45. Rajan, Diomede, Bramanti, et al. Conditioned medium from human gingival mesenchymal stem cells protects motor-neuron-like NSC-34 cells against scratch-injury-induced cell death. Int J Immunopathol Pharmacol. 2017; 30: 383-94.

46. Bakopoulou, Apatzidou, Aggelidou, et al. Isolation and prolonged expansion of oral mesenchymal stem cells under clinical-grade, GMPcompliant conditions differentially affects "stemness" properties. Stem Cell Res Ther. 2017; 8: 247.

47. Lee, Jeon, Kim, et al. Characteristics of stem cells from human exfoliated deciduous teeth (SHED) from intact cryopreserved deciduous teeth. Cryobiology. 2015; 71: 374-83.

48. Lindemann, Werle, Steffens, et al. Effects of cryopreservation on the characteristics of dental pulp stem cells of intact deciduous teeth. Arch Oral Biol. 2014; 59: 970-6.

49. Ginani, Soares, Rabelo, et al. Effect of a cryopreservation protocol on the proliferation of stem cells from human exfoliated deciduous teeth. Acta Odontol Scand. 2016; 74: 598-604. 